\title{
Notes
}

\section{The use of Kovar seals in high temperature gas chromatography with glass columns}

At present there appear to be two major methods for connecting glass columns to the carrier gas inlet tube and the cletector cell of gas chromatographs. In one, the column has socket ends which seat cylindrical silicone rubber septa. The gas enters or leaves the sockets through narrow steel tubes which penetrate the septa. The steel tubes are attached to the gas inlet tube or detector by means of metal compression fittings, usually Swagelok. In the second method, Swagelok metal fittings are used with Teflon ferrules or silicone O-rings to make direct connection with the glass column. Both methods have drawbacks when used at high temperatures, as the plastic connectors lose their elasticity and require periodic replacement. Depending on the nature of the physical stress in the connection system, these methods fail at temperatures as low as $220^{\circ}$. The most sensitive test of failure consists of comparing the peak areas of a standard mixture of early-eluting and late-eluting substances; the latter gives low areas when there is a leak.

We wish to point out the suitability of Kovar connections, which appear to be usable indefinitely at temperatures of $310^{\circ}$ or higher. A Kovar seal consists of a special stainless steel tube, sealed by a series of graded glasses to a borosilicate glass tube. Professional glassblowers make these in a range of sizes and some sizes are available commercially (Kontes Glass Co.). The metal part should be specified to be

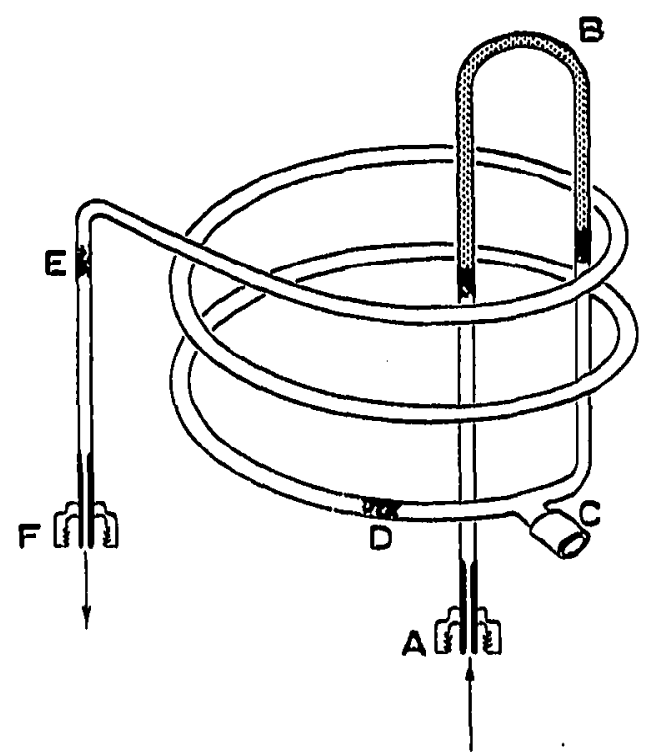

Fig. r. Novar column for gas chromatograph having two parallel metal fittings for gas inlet and outlet. $A=$ inlet Kovar seal with Swagelok nut; $B=$ reservoir column, packing with glass wool encls; $\mathrm{C}=$ injection socket (Burrell Corp.); $\mathrm{D}=$ beginning of column packing; $E=$ encl of column packing: $F=$ outlet Tiovar seal with Swagelok nut, to detector. 
at least $7 / 8$ in. long in order to accommodate the Swagelok nut and ferrules. The metal tubes are attached to the gas chromatograph fittings in the same way as with metal columns.

Although Swagelok fittings are described as being free of torque transmission, in our hands a certain amount of twisting occurs during tightening. Metal columns can withstand this twisting effect, but glass columns are somewhat more limited in this respect. We found it necessary to lubricate the Kovar tube, nut, and ferrules with Apiezon L grease before assembling the parts. (Such greasing would probably be helpful with metal columns too.) Disconnecting the column from the instrument calls for cautious use of narrow pliers to prevent torque transmission in the other direction.

One of our columns is attached to a Model 609 gas chromatograph (F \& M Scientific Corp.), the instrument's present $1 / 4$ in. gas inlet and outlet connectors being utilized. Fig. I shows the column, with some turns omitted in the sketch. The carrier gas enters the column through Kovar seal (A), then passes through a "reservoir column" (B), a short length of tubing packed with the same packing as the main column 1 .

The sample is injected through a silicone cylindrical septum held by a short socket tube (C). The packing is held in the column between points (D) and (E), and is loaded before the upper loop (B) is sealed onto the column. The carrier gas leaves the column via Kovar seal (F). Swagelok nuts are shown attached to the metal seals, but the ferrules are omitted from the drawing.

The septum used here is a newly formulated rubber (Applied Science Laboratories) which has a remarkably long life. The injection zone is heated by means of a length of resistance wire which is covered with asbestos cement and controlled by a transformer. Thus it is not necessary to use the metal injection block of the chromatograph.

Since this instrument uses an inverted pot as the oven cover, it is necessary to build a short insulating cylinder through which the septum and heating wires protrude and on which the pot sits.

Our other Kovar column is adapted to an oven which uses a flat septum for injection (Model 7oo, F \& M Scientific Corp.). The front end of the column, together with part of the injection hardware, is shown in Fig. 2. The Kovar seal (A) is attached

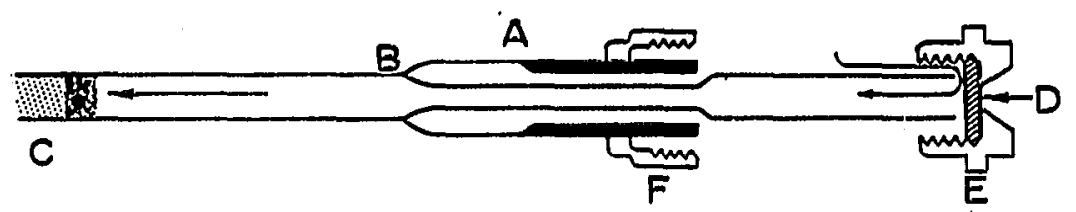

Fig. 2. Inlet system using Kovar seal and flat septum. A = Kovar seal and Swagelok nut, for attachment to metal injector; $\mathrm{B}=$ ring seal, attaching Kovar seal to glass column; $\mathrm{C}=$ beginning of column packing; $D=$ flat silicone septum; $E=$ retaining nut, to hold septum tightly against metal injector.

to the column by a ring seal at point (B). The front end of the column comes quite close to the silicone septum (D), which is held in place by the gas chromatograph's injector nut (E). The Kovar seal is attached at its metal end by a Swagelok nut (F) and ferrules (not shown). The two nuts $(E)$ and $(F)$ form a gas-tight seal to the heated 
injector by which carrier gas enters the column as shown. The back end of the column is connected directly to the instrument's Swagelok fitting by a simple Kovar seal, as in Fig. $I$.

The column in Fig. I includes the reservoir section because of the report by HUNTER AND WALDEN ${ }^{1}$ that this improves the column efficiency and longevity. We confirmed the improved efficiency in the case of poly(diethylene glycol succinate) but there was no effect with Apiezon $L$. We found that improved separations were obtained with both of the above packings when o.I $\mathrm{ml}$ of hexamethyldisilazane was slowly injected into the injection zone while the column was maintained at $225^{\circ}$. The reagent, which apparently reacts with $\mathrm{OH}$ groups in the column ${ }^{2}$, is eluted rather slowly and heating must be continued for several hours at elevated temperatures before samples can be injected.

Since making these columns, we have learned of another application of Kovar seals to glass column chromatography ${ }^{3}$. In this case the adaptation was intended to allow use of long biopsy needles. A basic difference in our applications is that CoHEN AND BREWER threaded their Kovar tube so that a flat silicone septum could be attached by means of a retaining nut (like (E) in Fig. 2). Their inlet and outlet connections are of the ordinary type, still subject to temperature limitations.

This work was supported in part by the U. S. Public Health Service research grant NB3Ig2 from the Institute of Neurological Diseases and Blindness.

Mental Health Research Institute, University of Michigan, Norman S. RadiN Ann Arbor, Mich. (U.S.A.)

I I. R. Hunter and M. Ir. Waidin, Anal. Chem., 35 (1963) I765.

2 J. Bohemen, S. H. Langer, IR. H. Perrett and J. H. Purneli, J. Chem. Soc., (1960) 2444. 3 E. N. Cohen aNd H. W. Brewer, J, Gas Chromatog., 2 (I964) 261.

Received June 2nd, I965

J. Chromatog., $20(1965) 392-394$

\section{Gas chromatography of alkaloids with polar stationary liquids}

\section{The effect of polyvinylpyrrolidone treatment of the support}

Coating of the solid support with polyvinylpyrrolidone (PVP) has been used to reduce adsorptive effects in gas chromatography of steroicls with polar stationary liquids ${ }^{1,2}$. The PVP treatment has also been shown to produce a preferential affinity of the column for alcohols, and to increase its selectivity for closely related plant sterols. Since adsorption by the support material is a major problem in quantitative gas chromatography of many alkaloids, the present investigation was undertaken to study the effect of PVP treatment. 